\title{
Einige typische Werte zum Wasserhaushalt der Schweizer Alpen, insbesondere des Kantons Tessin
}

In dieser vorläufigen Mitteilung sollen für einzelne alpine Untersuchungsgebiete hydrologische Bilanzen gemäß der Gleichung: Niederschlag = Abfluß + Verdunstung vorgelegt werden. Sie sind Arbeiten entnommen, die sinngemäß in der Fortsetzung des Pionierwerks von OTTO LÜTSCHG (1872-1947) stehen. Was Zitierung und alle weitern Detailangaben betrifft, sei verwiesen auf eine demnächst erscheinende umfassendere Publikation des Schreibenden über «Hydrologische Studien im zentralen schweizerischen Alpenvorland, insbesondere im Gebiet der Langete» (Beiträge zur Geologie der Schweiz - Hydrologie Nr. 22). Wie Tab. 1 zeigt, entstammen die Zahlen verschiedenen Autoren und Beobachtungsperioden; da es sich jedoch bei den letztern um langjährige Reihen handelt, sind Vergleiche zulässig.

Als Bilanz-Typen für mittlere Verhältnisse dürfen in Fig. 1 jene der Aare, Thun (Alpennordseite), der Verzasca, Corippo (Alpensüdseite, Sopraceneri) und der

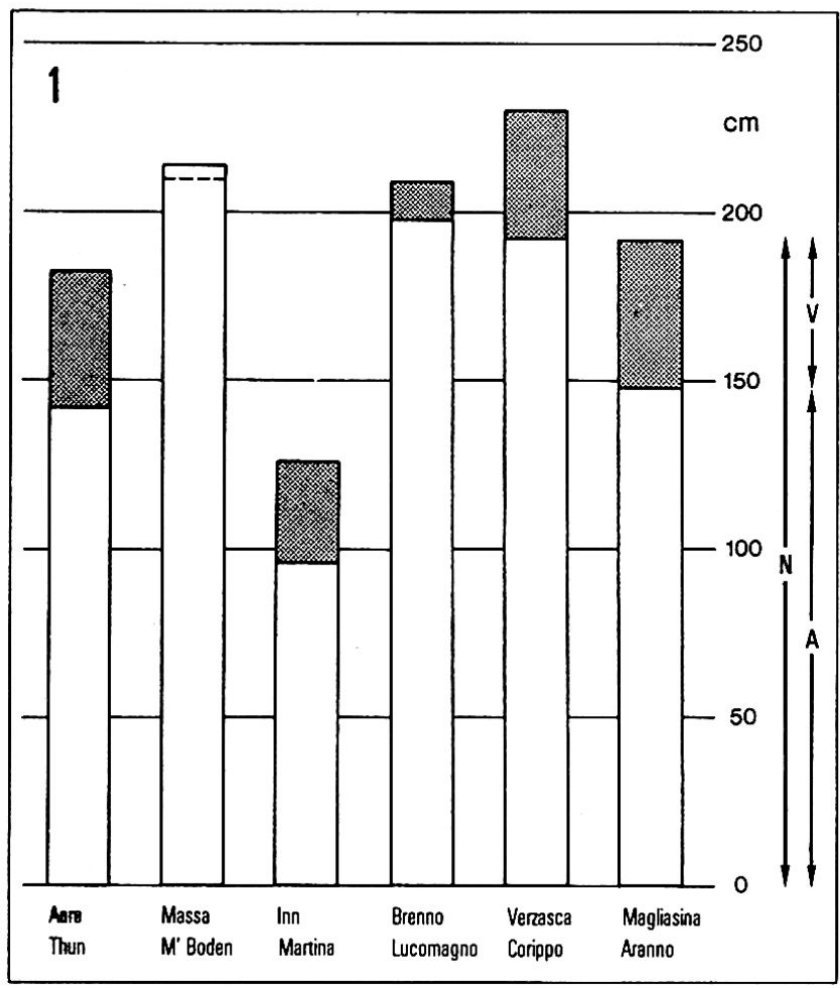

Fig. 1 Typische hydrologische Bilanzen einiger Untersuchungsgebiete der zentralen Alpen. Niederschlag $\mathrm{N}=$ Abfluß A + Verdunstung V. Mittlere Jahreswerte in $\mathrm{cm}$ gemäß Tabelle 1
Magliasina, Aranno (Alpensüdseite, Sottoceneri) gelten. Die bekannte Asymmetrie der Niederschlags- und Abflußmengen auf Alpennord- und Südseite wird erhellt durch einen Vergleich zwischen Aare, Thun und Verzasca, Corippo. Sehr ähnliche hydrologische Größen weisen die aus sehr ungleichen Höhenlagen stammenden Bilanzen von Aare und Magliasina auf. Einheitlich aber tritt für die Verdunstung der 3 Gebiete ein Wert von 380-440 mm auf (mittlere jährliche Landesverdunstung). Dies ist ein Hinweis mehr auf die alte These der «Verdunstung als Halbvariante» von FISCHER, 1936.

Als charakteristisch für ein inneralpines, überdies kontinental beeinflußtes Hochtal mag der Wasserhaushalt des Inns stehen, dessen tiefe Werte vorwiegend durch Niederschlagsschatten der Gebirgsumrahmung zu begründen sind. Der Anteil des vom Niederschlag zum Abfluß gelangenden Wassers (Abflußfaktor, Quotient von Abfluß/Niederschlag $=0.76$ ) stimmt dagegen größenordnungsmäßig wieder mit den vorgenannten Gebieten überein.

Ungewöhnliche Bilanzen liegen bei Massa und Lukmanierbrenno vor. Im hochalpinen, stark vergletscherten Gebiet der Massa übersteigt der Abfluß sogar den zeitlich zugehörigen Niederschlag, was durch die Gletscherschmelze ermöglicht wird. Wie weit hier noch Fehlbeträge auf Konto Niederschlag gehen - zufolge der sehr erschwerten Messung - können wir nicht ermessen. Jedenfalls sinkt hier die Verdunstung auf minimale Werte. - Rechnen wir andrerseits von den Abflußzahlen des karsthydrologisch regierten Brenno die Fremdwasserzuschüsse ab, entsprechen die Bilanzgrößen den allgemeinen Verhältnissen der Region des Sopraceneri.

Schließlich zeigt die Zusammenfassung von Fig. 2 (Ziffern teils in Tabelle) neben einer untern Gruppe von Mittellandflüssen (Kreise, 1-13) und einer mittlern der Alpennordseite (Quadrate, 20-43) den deutlich weitgestreuten Punktschwarm (Dreiecke, 53-58) der wasserreichen Tessiner Gebiete, die den obern Abschluß bilden. Es sind außer den in Tabelle 1 genannten, die folgenden:
53 Bavona, Bignasco
54 Rovana, Collinasca
55 Isorno, Russo
56 Brenno, Campo Blenio
58 Vedeggio, Isone

Dr. Valentin Binggeli, Brunnenrain 1, 4900 Langenthal BE 
Tabelle 1: Typische Bilanzen des Wasserhaushalts von Flußgebieten der Schweizer Alpen

\begin{tabular}{|c|c|c|c|c|c|c|c|c|c|}
\hline $\begin{array}{l}\text { Einzugsgebiet } \\
\text { Fluß, Abfluß- } \\
\text { station }\end{array}$ & $\begin{array}{l}\text { Zif. } \\
\text { in } \\
\text { Abb. } 2\end{array}$ & $\begin{array}{l}\text { Fläche } \\
\mathrm{km}^{2}\end{array}$ & $\begin{array}{l}\text { Glet- } \\
\text { scher } \\
\%\end{array}$ & $\begin{array}{l}\text { Höhe } \\
\text { m ü. M. }\end{array}$ & $\begin{array}{l}\text { Nieder- } \\
\text { schlag } \\
\mathrm{cm}\end{array}$ & $\begin{array}{l}A b- \\
\text { fluB } \\
\mathrm{cm}\end{array}$ & $\begin{array}{l}\text { Verdun } \\
\text { stung } \\
\mathrm{cm}\end{array}$ & $\begin{array}{l}\text { Mess- } \\
\text { per. }\end{array}$ & Autor \\
\hline Aare, Thun & 29 & 2490 & 10.9 & 1760 & 182 & 141 & 41 & $1930-55$ & WALSER, 1957 \\
\hline Massa, Massaboden & 42 & 202 & 67.6 & 2920 & 209 & 214 & 21 & $1931-70$ & KASSER, 1967 \\
\hline Inn, Martina & 70 & 1790 & 5.4 & 2350 & 126 & 96 & 30 & $1904-40$ & WALSER, 1967 \\
\hline Brenno, Campra & 52 & 35 & 0 & 1907 & 209 & 197 & - & $\begin{array}{l}1953 / 54- \\
1967 / 68\end{array}$ & BINGGELI, 1970 \\
\hline $\begin{array}{l}\text { Brenno, Campra } \\
\text { exkl. Fremdwasser }\end{array}$ & 52 & 35 & 0 & 1907 & 209 & 174 & 35 & s. oben & s. oben \\
\hline Verzasca, Corippo & 57 & 189 & 0 & 1680 & 230 & 192 & 38 & $\begin{array}{l}1956 / 57- \\
1963 / 64\end{array}$ & GRÜTTER, 1967 \\
\hline Magliasina, Aranno & 50 & 22 & 0 & 1055 & 192 & 148 & 44 & $1939-44$ & GYGAX, 1948 \\
\hline
\end{tabular}

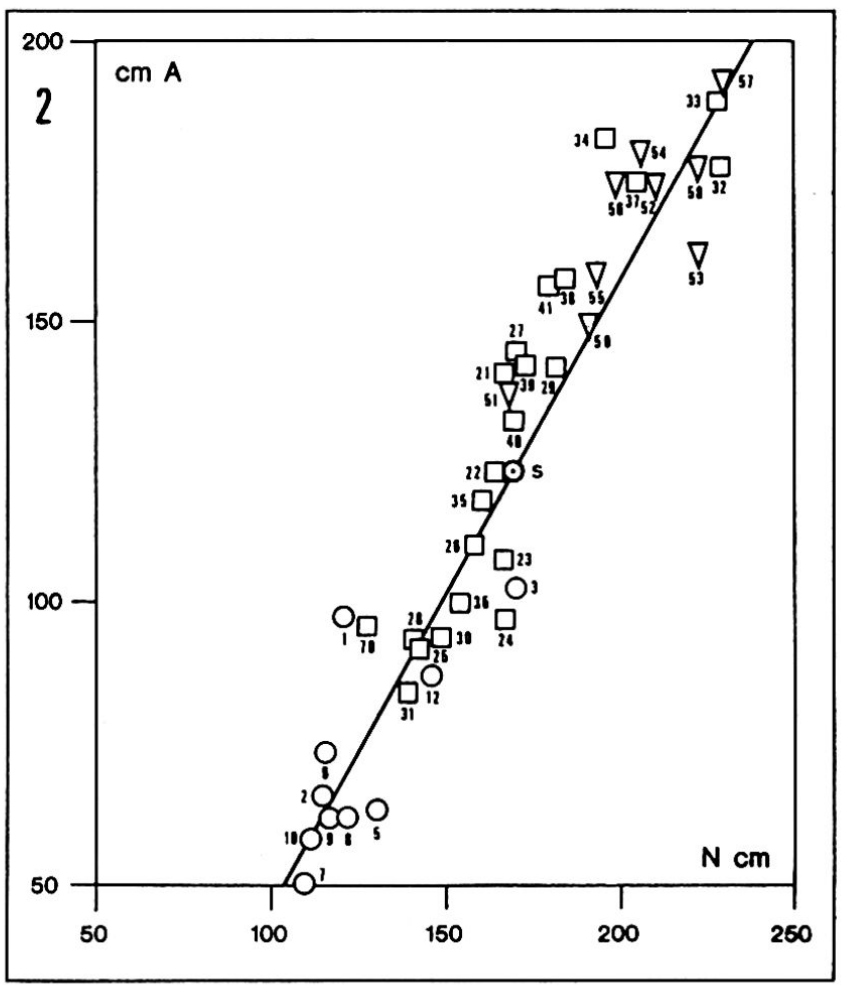

Fig. 2 Abflußfunktion für 40 schweizerische Flußgebiete: Abhängigkeit des Abflusses $A$ vom Niederschlag $N$. Regression: $A-1.114 \mathrm{~N}-65.13(\mathrm{~cm}) ; \mathrm{r}-0.94$. Die Schwerpunktskoordinaten der Ausgleisgeraden lauten: Niederschlag - $168.7 \mathrm{~cm}$; Abfluß $-122.8 \mathrm{~cm}$

\section{Literatur}

Es werden nur die direkt als Unterlagen, insbesondere zu Tabelle 1, Fig. 1 und 2, benützten Publikationen zitiert. Für alle weitern Angaben siehe in der eingangs angeführten Arbeit.

Amt für Wasserwirtschaft, Eidg. (p. a. seit 1917): Hydrographisches Jahrbuch der Schweiz. Bern

BINGGELI V. (1960): Karsthydrologische Wasserhärtestudien im Lukmaniergebiet. Mitt. Schweiz. Natf. Ges. Zürich

BINGGELI V. (1961): Zur Morphologie und Hydrologie der V. d. Lucomagno, Ticino. Beitr. z. Geol. d. Schweiz - Hydrologie 12. Bern

BINGGELI V. (1970): Niederschlagskarte und hydrologische Bilanz des Lukmaniertals. Mitt. Natf. Ges. Bern BURGER H. (1954): Einfluß des Waldes auf den Stand der Gewässer im Sperbel- und Rappengraben. Mitt. Eidg. Anstalt f. d. forstl. Vers.wesen. Zürich

BURGER H. (1945): Der Wasserhaushalt im V. d. Melera. Mitt. Eigd. Anstalt f. d. forstl. Vers.wesen. Zürich

FISCHER K. (1936): Ziele und Wege der Untersuchungen über den Wasserhaushalt. Mitt. deutsch. Wasserwirtsch. 40. Berlin

GRUUTTER E. (1967): Beiträge zur Morphologie und Hydrologie des V. Verzasca. Diss. Bern

GUILCHER A. (1965): Précis d'Hydrologie. Paris 
GYGAX F. (1948): Niederschlag und Abfluß im Einzugsgebiet der Magliasina. Beitr. z. Geol. d. Schweiz - Hydrologie 4. Bern

HIRSBRUNNER G. (1959): Morphologie und Hydrologie der Rovanatäler. Diss. Bern

International Hydrological Decade, Kommission on the (1968, 1972): Run-off Regimen an Water Balance I/II. Freib. Geogr. H. 6 und 12. Freiburg i. B.

JAGGI C. (1970): Hydrologische Untersuchungen in verschiedenen Tessiner Tälern. Diss. Bern

KASSER P. (1967): Hydrographie. In: Atlas der Schweiz III. Bern

KELLER R. (1961): Gewässer und Wasserhaushalt des Festlandes. Berlin

KISTLER E. (1954): Hydrologische Untersuchungen in der V. Onsernone. Diss. Bern

KRESSER W. (1961): Hydrologische Betrachtung der österreichischen Gewässer. Verh. Int. Ver. Limnol. XIV. Stuttgart

KRESSER W. (1973): Die Donau und ihre Hydrologie. Wasser- und Energiewirtschaft 3/4. Baden-Zürich
LÜTSCHG O. (1944-1954): Zum Wasserhaushalt des Schweizer Hochgebirges. Band I, II. Beitr. z. Geol. der Schweiz - Hydrologie 4. Bern

REIST M. (1960): Morphologie und Hydrologie des Bavonatales. Diss. Bern

RODER E. (1914): Niederschlag und Abfluß im bündnerischen Rheingebiet. Mitt. Abt. für Landeshydrographie Bern

WALSER E. (1954): Niederschlags- und Abflußverhältnisse im Einzugsgebiet des Rheins oberhalb von Basel. Wasser- und Energiewirtschaft 5-7. Zürich

WALSER E. (1957): Niederschlags- und Abflußverhältnisse im Einzugsgebiet der Aare. Wasser- und Energiewirtschaft 7-9. Zürich

WALSER E. (1958): Niederschlag und Abfluß im Reußgebiet. Wasser- und Energiewirtschaft 8/9. Zürich

WALSER E. (1967): Niederschlags- und Abflußverhältnisse im Engadin. Wasser- und Energiewirtschaft 6/7. Zürich

WITSCHI R. (1957): Morphologie und Hydrologie der oberen Bleniotäler. Diss. Bern

wUNDT w. (1953): Gewässerkunde. Berlin 